\title{
A grid-free least-squares method for pressure evaluation from LPT data
}

\author{
M. Bobrov ${ }^{1,2}$, M. Hrebtov ${ }^{1,2}$, V. Ivashchenko ${ }^{1}$, R. Mullyadzhanov ${ }^{1,2}$,

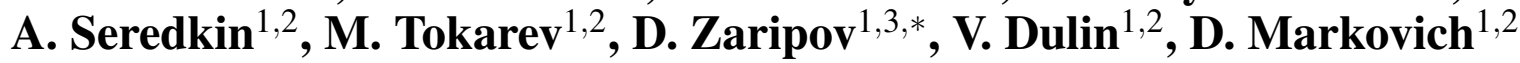 \\ ${ }^{1}$ Institute of Thermophysics SB RAS, Lavrentyev ave. 1, 630090 Novosibirsk, Russia \\ ${ }^{2}$ Novosibirsk State University, Pirogov str. 2, 630090 Novosibirsk, Russia \\ ${ }^{3}$ School of Mechanical Science and Engineering, Huazhong University of Science and Technology, \\ 430074 Luoyu Road 1037, Wuhan, China \\ *zaripov.d.i@gmail.com
}

Lagrangian particle tracking Shake-the-box (STB) method (Schanz et al., 2016) acquires the 3D positions of tracer particles from the temporal sequences of their 2D projection images even for rather high seeding densities. Approximation of tracks by analytical functions (Gesemann, 2015) provides an accurate evaluation of tracers' local velocity and acceleration. This data, which is obtained on non-regular grid, can be used to estimate local pressure fluctuations based on the Navier-Stokes equation. The present paper describes a grid-free least-squares method for the gradients and pressure evaluation based on irregularly scattered LPT data with random noise minimization.

The performance of the employed method is assessed based on synthetic images of virtual particles seeded in a wall-bounded turbulent flow. The tracks are obtained from the direct numerical simulation (DNS) of an initially laminar boundary layer flow around a hemi-sphere, mounted onto a flat wall. The Reynolds number based on the sphere diameter and free stream velocity is 7000 corresponding to a fully turbulent wake (see Figure 1).

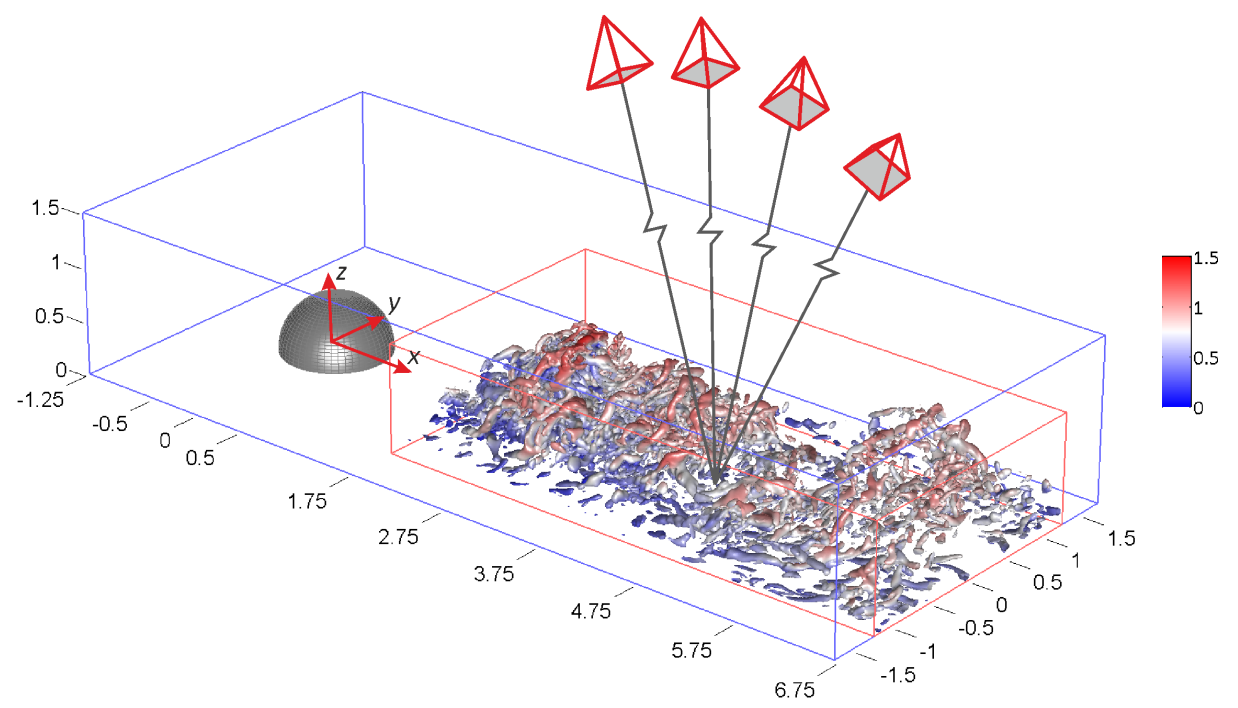

Figure 1: Location of the virtual cameras with respect to the coordinate system and a flow domain where particles were initially seeded (larger box) and ROI (smaller box) with visualization of the vortex structures with instantaneous isosurface of $\lambda_{2}=-5$ colored by streamwise velocity magnitude

In order to study the effect of particle image concentration (up to $0.2 \mathrm{ppp}$ ) on the overall accuracy, two hundred thousand inertial particles were randomly and uniformly seeded into a flow domain as illustrated 
in Figure 1. We considered four concentrations, i.e. $N_{p p p}=0.005,0.05,0.12$ and 0.2 ppp. To quantify the image noise effect, a random noise with different amplitude was added to the projection images for the moderate case of $N_{p p p}=0.05$. The images were processed using an STB algorithm (similar to that of Gesemann) enabling an accurate tracking of particles and evaluation of their velocity and acceleration. The firstand second-order gradients of the velocity and acceleration were evaluated using a grid-free least-squares method (Mavriplis, 2003). Afterwards the local values of pressure were computed employing a modified version of the method, where the pressure gradients were obtained directly from the Navier-Stokes equations (Kuhnert and Tiwari, 2004). Besides, in order to reveal the effect of spatial resolution and performance of the employed algorithms, the original tracks from the DNS were used to evaluate the same flow quantities. We refer to such case as Hacker's data.

The examples of the results are presented in Figures 2 and 3 . Particle image concentration $N_{p p p}=0.05$ is shown in Figure 2 for the comparison with the Hacker's data. The velocity and acceleration field are interpolated to the regular grid using the least-squares method, whereas the pressure is evaluated from the Poison equation. The resolution is sufficient to recover large-scale flow structures and negative pressure regions in the wake.

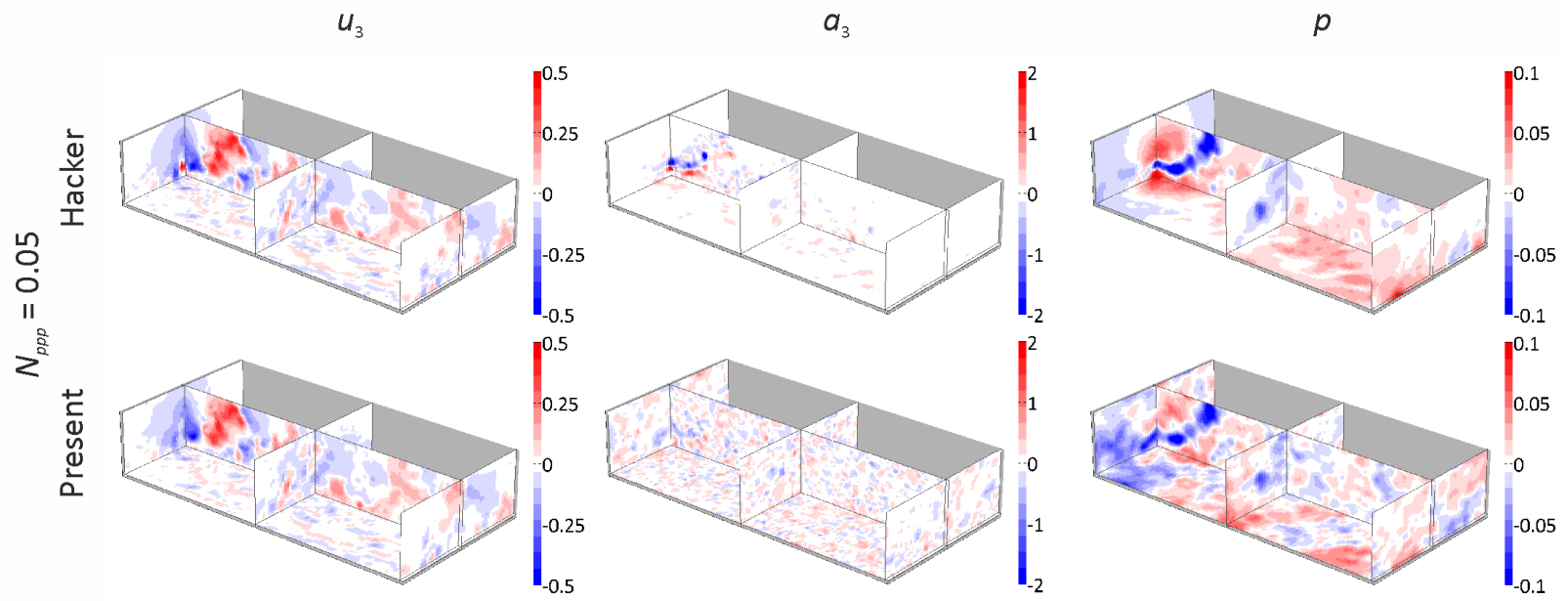

Figure 2: The instantaneous distributions of the wall-normal velocity (left) and acceleration (middle) components and pressure (right) obtained for Hacker's and STB data
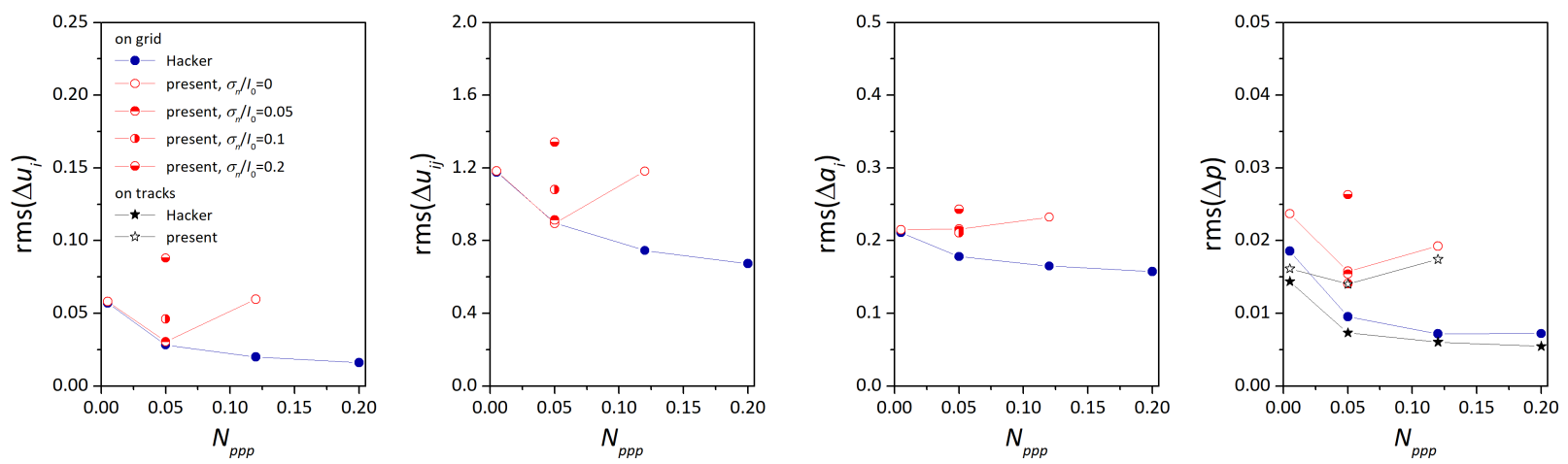

Figure 3: The root-mean-square deviations of the error between the exact and estimated velocities, velocity gradients, accelerations and pressure (from left to right) for different concentrations and image noise level for $N_{p p p}=0.05$

The performance of the used STB algorithm is limited for the cases of high seeding density $N_{p p p}=0.12$ and noise level of $10 \%$. Besides, the spatial resolution is not enough to approximate accurately the velocity gradients and particles acceleration on the regular grid. However, the least-squares method demonstrates a 
remarkable accuracy for the pressure evaluation from the exact particles' locations, velocities and acceleration (see Hacker's data in Figure 3), viz., even for $N_{p p p}=0.05$ the pressure error is below $10 \%$.

\section{Acknowledgements}

This work was financially supported by the Russian Science Foundation, grant number 19-79-30075.

\section{References}

Gesemann S (2015) From particle tracks to velocity and acceleration fields using b-splines and penalties. arXiv preprint arXiv: 151009034

Kuhnert J and Tiwari S (2004) Grid free method for solving the poisson equation. Wavelet Analysis and Applications pages 151-166

Mavriplis D (2003) Revisiting the least-squares procedure for gradient reconstruction on unstructured meshes. in 16th AIAA Computational Fluid Dynamics Conference, Orlando, USA, June 23-26, 3986 $1-13$.

Schanz D, Gesemann S, and Schröder A (2016) Shake-the-box: Lagrangian particle tracking at high particle image densities. Experiments in fluids 57:1-27 